unfavorable outcome, while 7 died. At a cutoff of 3, STEPSS had $93.3 \%$ sensitivity, $80.8 \%$ specificity, and negative predictive value of $99 \%$ for unfavorable outcome. The negative predictive values for death and treatment failure (refractory or super-refractory status epilepticus) were $99 \%$ and $98 \%$, respectively. At a cutoff of 2 , STEPSS had $100 \%$ sensitivity, $60.6 \%$ specificity for unfavorable outcome, and 100\% negative predictive value for unfavorable outcome, death, and treatment failure. The predictive accuracy was comparable to that of STESS in adults.

Conclusion: The STEPSS, a simple bedside clinical score was found to be useful to predict the outcome and treatment response in children with status epilepticus.

\section{A0042: Role of Pyridoxal Phosphate in Modulation of Endogenous Kynurenic Acid Synthesis Associated with Hippocampal Sclerosis}

Soumil Dey, ${ }^{1}$ Aparna Dixit, ${ }^{1}$ Manjari Tripathi, ${ }^{1}$ P. Sarat Chandra, ${ }^{1}$ Jyotirmoy Banerjee ${ }^{1}$

${ }^{1}$ Center of Excellence for Epilepsy, A Collaborative Project between All India Institute of Medical Sciences, New Delhi, National Brain Research Center, Manesar, India

Objective: Hippocampal sclerosis (HS) is the most common form of drug-resistant epilepsy where temporal lobe structures are responsible for unprovoked seizures. The hallmark of HS is enhanced glutamatergic excitatory neurotransmission. Pyridoxal 5-phosphate $(p L P)$ is an active form of vitamin B6 and plays an important role as a cofactor of various enzymes including kynurenine aminotransferase II (KAT II), which catalyzes kynurenic acid (KYNA) synthesis from kynurenine within cortical astrocytes. KYNA, a tryptophan metabolite, is the only natural inhibitor of glutamate receptors. The present study was designed to test the hypothesis that in HS, altered PLP concentration is responsible for reduced endogenous kynurenic acid synthesis in hippocampus.

Methods: Hippocampus from HS patients $(n=33)$ were used for this study. Tissues resected from tumor margin during brain tumor surgery of seizure-free patients as nonepileptic control $(n=14)$ were used. To determine total KYNA and PLP concentration, tissues were kept in perchloric acid at $-80^{\circ} \mathrm{C}$. Tissues were homogenized, centrifuged, and supernatants were collected and estimated using HPLC with fluorescence detection ( $p$ LP excitation 300 nm, emission 400 nm; KYNA excitation 344 nm, and emission 404 nm).

Results: KYNA concentration was significantly less in HS hippocampus $(0.0244 \pm 0.0219 \mathrm{ng} / \mathrm{mg}$ of protein $)$ compared with nonepileptic controls $(0.2117 \pm 0.1332 \mathrm{ng} / \mathrm{mg}$ of protein). PLP concentration was also significantly less in HS patients (1.77 $\pm 3.96 \mathrm{mg} / \mathrm{mg}$ of protein) compared with controls (10.91 $\pm 11.34 \mathrm{ng} / \mathrm{mg}$ of protein).

Conclusion: PLP concentration is reduced which, we suspect, may consequences in decreased KYNA due to dysfunctional machinery to synthesize kynurenic acid from kynurenine in HS patients.

Acknowledgment This work was supported by the Centre of Excellence for Epilepsy, a collaborative project between All India Institute of Medical Sciences, New Delhi, and National Brain Research Centre, Manesar; and funded by Department of Biotechnology, Ministry of Science and Technology, Govt. of India (Grant: BT/01/COE/09/08).

\section{A0043: A Home-Based, Primary-Care Model for Epilepsy Care in Low and Middle Income Countries: Basis and Design}

Suman Sharma, ${ }^{1}$ Anuraag Chowdhury, ${ }^{1}$ Sarit Sharma, ${ }^{1}$ Rajinder Bansal, ${ }^{1}$ Gagandeep Singh ${ }^{1}$

${ }^{1}$ Department of Neurology, Dayanand Medical College, Ludhiana, Punjab, India

Objective: A cluster-randomized trial of home-based care using primary care resources for people with epilepsy has been set up to optimize epilepsy care in resource-limited communities in low- and middle- income countries. The primary aim is to determine whether treatment adherence to antiepileptic drugs is better with home-based care or with routine clinic-based care. Secondary aims are to compare the effects of the two care pathways on seizure control and quality of life.

Methods: The home-based intervention comprises epilepsy medication provision, adherence reinforcement, and epilepsy self-management and stigma management guidance provided by an auxiliary nurse-midwife equivalent. The experimental group will be compared with a routine clinic-based care group using a cluster-randomized design in which the unit of analysis is a cluster of 10 people with epilepsy residing in an area cared for by a single accredited government grass-root health worker. The primary outcome is treatment adherence as measured by monthly tablet counts and two self-completed questionnaires. The secondary outcomes include monthly seizure-frequency, time to first seizure (in days) after enrolment, proportion of subjects experiencing seizure freedom for the duration of the study and quality of life measured by the "Personal Impact of Epilepsy Scale," all assessed by an independent study nurse.

Results: The screening phase and neurological evaluations and randomizations have been recently completed and follow-up is currently underway.

Conclusion: The Results of the trial are likely to have substantial bearing on the development of governmental policies and strategies to provide coverage and care for epilepsy in resource-limited countries.

\section{A0044: Clinical and Demographic Profile of Epilepsy Patients in Rural Rajasthan \\ Surbhi Chaturvedi, ${ }^{1}$ R.K Sureka, ${ }^{1}$ Amit Agarwal ${ }^{1}$ \\ ${ }^{1}$ Mahatma Gandhi Medical College and Hospital, Jaipur, Rajasthan, India}

Objective: The present study attempted to explore the clinical and demographic profile of epileptic patients, and pharmacological management in these patients.

Methods: The prospective study was done in center situated at Ratannagar, a Community Health Centre in district Churu under the auspicious of Epilepsy Care and Research foundation, an NGO involved with epilepsy work. It included 6,993 epileptic patients who visited the center from majority 